\title{
Quantitative imaging of airway liquid absorption in cystic fibrosis
}

\author{
Landon W. Locke1, Michael M. Myerburg ${ }^{1}$, Matthew R. Markovetz², \\ Robert S. Parker², Lawrence Weber ${ }^{3}$, Michael R. Czachowski ${ }^{4}$ Thomas J. Harding ${ }^{4}$, \\ Stefanie L. Brown ${ }^{1}$, Joseph A. Nero' ${ }^{2}$, Joseph M. Pilewski ${ }^{1,5}$ and \\ Timothy E. Corcoran ${ }^{1}$
}

Affiliations: 'Division of Pulmonary, Allergy, and Critical Care Medicine, University of Pittsburgh, Pittsburgh, PA, USA. ${ }^{2}$ Dept of Chemical Engineering, University of Pittsburgh, Pittsburgh, PA, USA. ${ }^{3}$ Dept of Radiology, University of Pittsburgh Medical Center, Pittsburgh, PA, USA. "Dept of Radiology, Children's Hospital of Pittsburgh, University of Pittsburgh Medical Center, Pittsburgh, PA, USA. ${ }^{5}$ Dept of Cell Biology, University of Pittsburgh, Pittsburgh, PA, USA.

Correspondence: Timothy E. Corcoran, University of Pittsburgh, UPMC MUH NW628, 3459 Fifth Ave, Pittsburgh, PA 15213, USA. E-mail: corcorantedupmc.edu

ABSTRACT New measures are needed to rapidly assess emerging treatments for cystic fibrosis (CF) lung disease. Using an imaging approach, we evaluated the absorptive clearance of the radiolabeled small molecule probe diethylene triamine penta-acetic acid (DTPA) as an in vivo indicator of changes in airway liquid absorption.

DTPA absorption and mucociliary clearance rates were measured in 21 patients with CF (12 adults and nine children) and nine adult controls using nuclear imaging. The effect of hypertonic saline on DTPA absorption was also studied. In addition, in vitro studies were conducted to identify the determinants of transepithelial DTPA absorption.

CF patients had significantly increased rates of DTPA absorption compared with control subjects but had similar mucociliary clearance rates. Treatment with hypertonic saline resulted in a decrease in DTPA absorption and an increase in mucociliary clearance in 11 out of 11 adult CF patients compared with treatment with isotonic saline. In vitro studies revealed that $\sim 50 \%$ of DTPA absorption can be attributed to transepithelial fluid transport. Apically applied mucus impedes liquid and DTPA absorption. However, mucus effects become negligible in the presence of an osmotic stimulus.

Functional imaging of DTPA absorption provides a quantifiable marker of immediate response to treatments that promote airway surface liquid hydration.

○

@ERSpublications

Functional imaging detects liquid absorption and mucociliary clearance in cystic fibrosis airways http://ow.ly/uPuX2

This article has supplementary material available from erj.ersjournals.com

Received: Dec 172013 | Accepted after revision: March 192014 | First published online: April 172014

Clinical trial: This study is registered at www.clinicaltrial.gov with identifier numbers NCT01223183 and NCT01486199.

Support statement: This project was supported by US National Institutes of Health grants NIH K25 HL08153, NIH R01 HL112863, NIH P30 DK072506 and NIH R01 HL108929, and a Cystic Fibrosis Foundation RDP to the University of Pittsburgh.

Conflict of interest: Disclosures can be found alongside the online version of this article at erj.ersjournals.com

Copyright (CERS 2014 


\section{Introduction}

Cystic fibrosis (CF) is a life-shortening autosomal recessive disease that affects $>70000$ people worldwide. It is caused by mutations in the cystic fibrosis transmembrane conductance regulator (CFTR) gene, which encodes a protein that functions as a chloride channel found in several organs. Alterations in the CFTR gene markedly impair ion conductance at the epithelial surface, which causes airway surface liquid (ASL) volume depletion and subsequent mucociliary clearance (MCC) dysfunction, infection and premature respiratory failure [1]. Recently, therapies that pharmacologically correct CFTR function within specific genotypebased patient subgroups have emerged [2, 3]. However, quantitative methods for assessing basic lung disease pathophysiology are needed to fully support the development and dissemination of these therapies.

Currently available outcome measures track the downstream effects of CF lung disease and, as such, do not provide a rapid indication of therapeutic response. Biomarkers such as nasal potential difference [4] and sweat chloride concentration can be useful for evaluating the basic efficacy of new treatments, but results obtained from these tests may not correlate with changes occurring in the lung. Pulmonary imaging methods can accelerate therapeutic development by mediating the rapid screening of new therapies and therapy combinations as well as dosing regimens in limited patient populations.

Liquid hyperabsorption is a key element of CF lung pathophysiology that cannot be measured directly through any currently available in vivo method. Aerosol-based pulmonary imaging methods have been developed to measure absorption through the airway epithelium [5] and MCC, the latter of which has been used to demonstrate the efficacy of inhaled osmotic therapies such as hypertonic saline in CF subjects $[6,7]$. The present investigation considers the use of a soluble, hydrophilic probe (diethylene triamine penta-acetic acid (DTPA)) as a surrogate marker of liquid absorption in the airways. In vivo, DTPA clearance includes both absorptive and mucociliary components. The latter can be estimated based on the clearance of a nonabsorbable, radiolabelled particle, and DTPA absorption then calculated. The clearance rate of the particle provides an independent measurement of MCC in the lung, a useful second outcome measurement. The rationale for our approach derives from cell culture studies showing that CF human bronchial epithelial (HBE) cells absorb liquid more rapidly than non-CF cells [8,9]. Previous in vitro results from our laboratory have shown that the absorption rate of radiolabelled DTPA is: 1) increased in CF HBE cell cultures compared with non-CF cell cultures; 2) well correlated with the ASL absorption rate; and 3) influenced by transepithelial osmotic gradients [10]. In vivo, we have previously shown that the absorptive clearance of DTPA is increased in the lungs of CF subjects compared with healthy volunteers [11]. Taken together, these results support the hypothesis that DTPA absorption correlates with transepithelial liquid absorption. The purpose of this study was to measure the absorptive lung clearance of DTPA in adult and paediatric CF and adult control subjects, and to assess DTPA absorption response in vivo following treatment with nebulised hypertonic saline.

\section{Methods}

Detailed description of materials and methods can be found in the online supplementary material.

\section{Subjects}

Imaging was performed in adult $\mathrm{CF}(\mathrm{n}=20)$, adult control $(\mathrm{n}=10)$ and paediatric $\mathrm{CF}(\mathrm{n}=10)$ patients. In a pre-determined, randomised order, the adult CF subjects received hypertonic saline (7\% sodium chloride) or isotonic saline $(0.9 \%$ sodium chloride) on separate study days. The paediatric CF and control groups performed only one study day (isotonic saline treatment). The study was not blinded. Written informed consent was obtained from all subjects. This study was approved by the University of Pittsburgh Institutional Review Board (IRB) (Pittsburgh, PA, USA). This study is registered at www.clinicaltrial.gov with identifier numbers NCT01223183 and NCT01486199.

\section{Inhalation of radiopharmaceuticals}

Radioaerosol delivery was achieved with a DeVilbiss 646 jet nebuliser (DeVilbiss Healthcare, Somerset, PA, USA) containing $55.5 \mathrm{MBq}$ indium-111-DTPA and $296 \mathrm{MBq}$ technetium-99m-sulfur colloid (SC) in 3-4 mL normal saline with breathing patterns and delivery techniques shown to provide predominantly airway deposition $[12,13]$.

\section{Imaging protocol}

An 80-min image acquisition ( $1 \mathrm{~min}$ per frame) was initiated immediately after aerosol delivery. At the start of the 11th frame $(10 \mathrm{~min})$ subjects inhaled either isotonic or hypertonic saline for $10 \mathrm{~min}$. Independent energy windows were used to image technetium-99m and indium-111. The 80-min imaging period included a 10-min baseline measurement, $10 \mathrm{~min}$ imaging during saline delivery and then $60 \mathrm{~min}$ continuous imaging. The 60-min imaging period is similar to previous measurements of MCC [14, 15]. 
Image data analysis

Right lung retention curves corrected for decay, background and isotope cross-talk contamination were image-derived for each radioisotope using a semiautomated software program written in house in MATLAB (Mathworks, Natick, MA, USA), which calculated the per cent clearance at $80 \mathrm{~min}$ of ${ }^{99 \mathrm{~m}} \mathrm{Tc}$-SC (referred to as MCC) and ${ }^{111}$ In-DTPA. The difference between MCC and total DTPA clearance is our estimate of DTPA clearance by absorption (ABS). These measures were also obtained from central lung zone retention curves. Additionally, the area above the whole-lung retention curve (AAC) was calculated for ${ }^{99 \mathrm{~m}} \mathrm{Tc}$-SC (AACTc) and ${ }^{111}$ In-DTPA (AACIn) by integration over all 80 frames. The difference between AACTc and AACIn is our estimate of the AAC for DTPA absorption (AACABS). The study analyst was not blinded but the opportunity for operator bias was negligible due to the automated nature of the analysis software.

\section{Primary HBE cell culture studies}

Primary HBE cells were isolated from excess lung airway tissue removed for transplantation under protocols approved by the University of Pittsburgh IRB as previously described [16].

\section{Effects of transepithelial fluid flow}

To determine the relative contributions of diffusion and convection on DTPA transport across the epithelium in CF HBE cell cultures, liquid absorption was blocked through the apical delivery of DTPA in a salt-free, iso-osmolar solution of mannitol in water. Unlike salt, HBE cells are unable to actively transport mannitol across their membranes [17], effectively impeding absorption of the liquid bolus. Using three different CF cell lines (six filters per line), radioactive counts and ASL volume [18] were measured over a 10 -h period starting at $2 \mathrm{~h}$ after the apical addition of $10 \mu \mathrm{L}$ radiolabelled DTPA in either normal saline or osmolarity-matched mannitol in water.

\section{Effects of mucus}

Excessive mucus accumulation is a signature of CF lung disease and previous studies have described DTPA binding to mucus [19]. To study its effects, radioactive counts and ASL volume were measured over $12 \mathrm{~h}$ following the addition of $10 \mu \mathrm{L}$ radiolabelled DTPA in normal saline to CF HBE cell cultures with or without added apical mucus. These measurements were repeated in the presence of a strong transepithelial fluid flow mediated by a hyperosmotic basolateral media $\left(600 \mathrm{mOsm} \cdot \mathrm{L}^{-1}\right)$. Two CF lines were tested (six filters per line).

\section{Statistical analysis \\ In vivo statistical analysis}

Central to peripheral deposition ratio (C/P), MCC, total DTPA clearance, ABS, and AACTc, AACIn and the difference between them were compared between patient groups by t-tests. A paired t-test was used to assess changes between the isotonic and hypertonic saline treatment study days. A series of independent linear regressions was performed to compare image-derived measurements with clinical variables.

In vitro statistical analysis

ASL volume and DTPA absorption rate measurements were compared by t-tests. The Mann-Whitney ranksum test was used to compare baseline rates of ASL volume and DTPA absorption to rates measured in the presence of mucus, hyperosmotic basolateral media or the combination of both. Sigma-Stat v2.0 (SPSS, Inc., Chicago, IL, USA) was used for all statistical testing.

\section{Results}

\section{Subject characteristics}

Age and lung function data from the three study groups are presented in table 1.20 adult CF subjects were enrolled. Four early subjects were excluded due to low deposited doses (technetium-99m $<500$ counts per min above background). The dosing period was increased at the midpoint of the study and all subsequent deliveries provided sufficient dosing. Two additional adult CF subjects were excluded due to nebuliser or camera malfunctions. Two adult CF patients were G551D heterozygous and on ivacaftor therapy. Because this new therapy is believed to have significant effects on lung physiology, their data are presented but not included in statistical comparisons. Therefore, data from 12 adult CF subjects were included in the primary study analysis with 11 subjects completing both hypertonic and isotonic saline treatment days. 10 of the 12 subjects were homozygous for the F508del mutation, one subject was heterozygous for the G551D mutation (not on ivacaftor) and one subject had multiple polymorphisms (7T/M470V-5T/M470V). 10 paediatric CF subjects and 10 healthy adult control subjects were also enrolled. One subject from each group was excluded due to inadequate lung deposition. Eight of the nine paediatric CF patients were homozygous for the 
TABLE 1 Subject pulmonary function and age

\section{Adult CF}

Subjects $\mathrm{n}$

FEV $1 \%$ predicted

FVC \% predicted

FEF25-75\% \% predicted

Age years mean $\pm S D$
14

$67.6 \pm 16.8(43-98)$

$81.7 \pm 18.9(56-120)$

$43.3 \pm 17.3(20-70)$

$23.9 \pm 5.5$
Paediatric CF

$90.1+14.5(62-106)$

$97.6 \pm 8.1(81-110)$

$79.8+28.9(45-134)$

$10.7 \pm 1.5$
Controls

$99.2+8.8(83-113)$

$102.9 \pm 6.4(94-114)$

$86.7 \pm 18.3(60-117)$

$32.2 \pm 15.5$

Data are presented as mean \pm SD (range), unless otherwise stated. CF: cystic fibrosis; FEV1: forced expiratory volume in 1 s; FVC: forced vital capacity; FEF25-75\%: forced expiratory flow rate at $25-75 \%$ of FVC.

F508del mutation and one patient was heterozygous for the nonsense mutation R1162X. More specific data on subject genotype and age are included in the online supplementary material.

\section{Composite retention data}

Mean normalised retention curves of indium-111 and technetium-99m in the whole right lung for each study group are shown in figure 1. Qualitatively, the adult CF patients (fig. 1a) demonstrated similar baseline MCC, total DTPA clearance and ABS to that of paediatric CF patients (fig. 1b). Inhaled hypertonic saline treatment increased MCC and total DTPA clearance in the adult CF cohort (fig. 1c) and decreased ABS to a value similar to that observed in control subjects (fig. 1d). Although not statistically significant, a gradually increasing difference in mean normalised ${ }^{99 \mathrm{~m}} \mathrm{Tc}$-SC clearance over $80 \mathrm{~min}$ was seen between the control and adult CF group (fig. 2).

\section{Image-derived measurements}

The average MCC in the adult CF, paediatric CF and control group was $23.6 \pm 10.3 \%, 23.0 \pm 13.9 \%$ and $31.4 \pm 13.1 \%$, respectively (nonsignificant by individual t-test between all groups) (fig. $3 a$ ). The mean \pm SEM
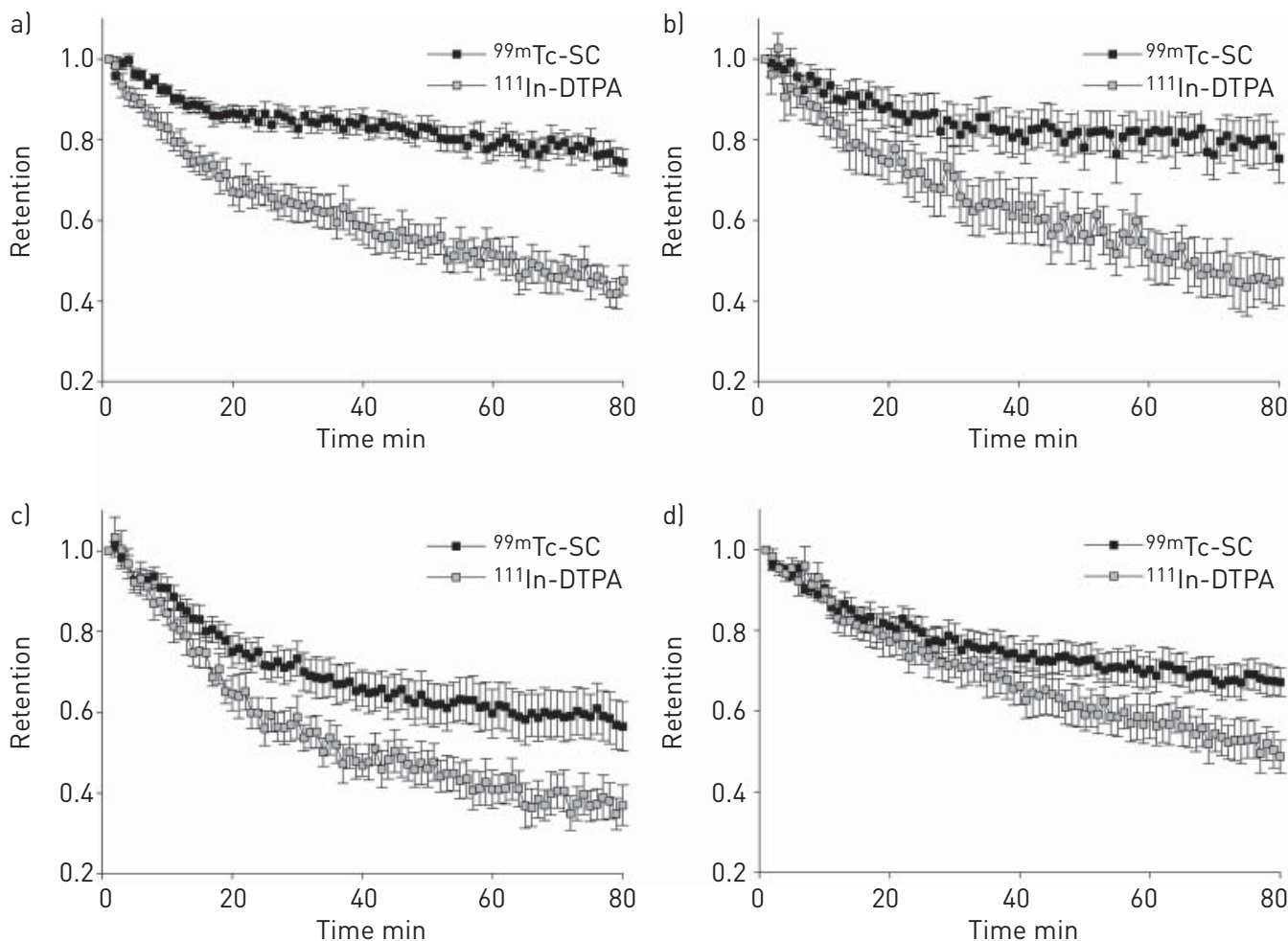

FIGURE 1 Normalised right lung retention of technicium-99m-sulfur colloid (SC) and indium-111- diethylene triamine penta-acetic acid (DTPA) plotted against time for the a) adult cystic fibrosis (CF) group $(n=12), b)$ paediatric CF group $(n=9), c)$ adult CF group with hypertonic saline treatment $(n=11)$ and $d)$ control group $(n=9)$. Data are presented as mean \pm SEM. 
FIGURE 2 Comparison of mean \pm SEM normalised technetium-99m-sulfur colloid (SC) retention curves in healthy volunteers $(n=9)$ and adult cystic fibrosis $(\mathrm{CF})(\mathrm{n}=12)$ subjects following nebulised isotonic saline treatment. The difference in per cent clearance at $80 \mathrm{~min}$ did not reach statistical significance $(\mathrm{p}=0.14)$.

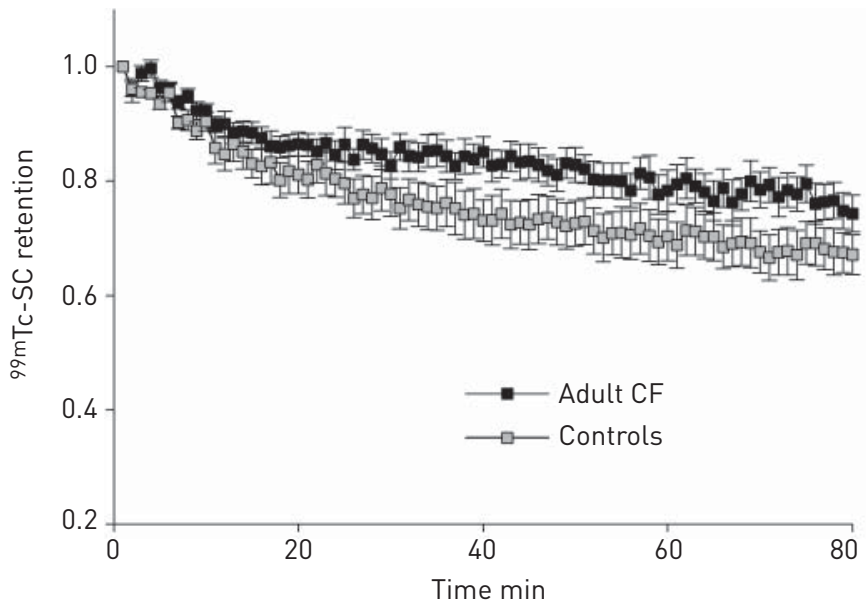

total DTPA clearance in the adult CF, paediatric CF and control group was $55.4 \pm 12.3 \%, 56.6 \pm 16.5 \%$ and $49.1 \pm 13.6 \%$, respectively (nonsignificant between all groups) (fig. $3 \mathrm{~b}$ ). The absorptive component of ${ }^{111}$ In-DTPA clearance is calculated for each subject by subtracting the ${ }^{99 \mathrm{~m}} \mathrm{Tc}$-SC clearance from the total ${ }^{111}$ In-DTPA clearance. The average ABS for the adult CF, paediatric CF and control group was $32.0 \pm 13.9 \%, 33.6 \pm 9.7 \%$ and $17.7 \pm 6.9 \%$, respectively (adult CF versus control: $p=0.010$; paediatric CF versus control: $\mathrm{p}=0.002$; adult $\mathrm{CF}$ versus paediatric $\mathrm{CF}$ : nonsignificant) (fig. $3 \mathrm{c}$ ). The two subjects with a G551D mutation on ivacaftor therapy had MCC values of $34.3 \%$ and $27.2 \%$, and ABS values of 15.7 and $11.2 \%$.

Central lung zone (large airway) measurements are reported in table 2. Similar to whole-lung analysis, the average MCC and total DTPA clearance were not statistically different between any of the three subject groups. Central lung zone ABS was significantly higher in the adult and paediatric CF groups compared with the control group (adult CF versus control: $p=0.014$; paediatric CF versus control: $p=0.003$; adult $C F$ versus paediatric $\mathrm{CF}$ : nonsignificant), which mirrored the findings of whole-lung analysis. AAC analysis (table 2) revealed no significant differences in AACTc or AACIn between any of the subject groups. However, AACABS was significantly higher in the adult and paediatric CF groups compared with the control group (adult CF versus control: $\mathrm{p}=0.010$; paediatric CF versus control: $\mathrm{p}=0.006$; adult $\mathrm{CF}$ versus paediatric CF: nonsignificant).

Mean $\mathrm{C} / \mathrm{P}$ in the $\mathrm{CF}$ adult, $\mathrm{CF}$ paediatric and control groups was $2.2 \pm 0.5,2.1 \pm 0.4$ and $2.4 \pm 0.3$, respectively ( $\mathrm{p}=0.18$ by ANOVA), providing an indication of predominantly bronchial airway deposition and uniformity of deposition pattern across all groups.

\section{Response to osmotic therapy in CF adults}

All 11 adult CF subjects showed a decrease in ABS and an increase in MCC following inhalation of hypertonic saline compared with isotonic saline treatment (ABS: $32.0 \pm 13.9 \%$ versus $22.2 \pm 12.8 \%$, $\mathrm{p}<0.001$; MCC: $23.6 \pm 10.3 \%$ versus $42.4 \pm 18.2 \%, \mathrm{p}=0.003$ ) (fig. $4 \mathrm{a}$ and $\mathrm{b}$ ). Six out of 11 subjects showed
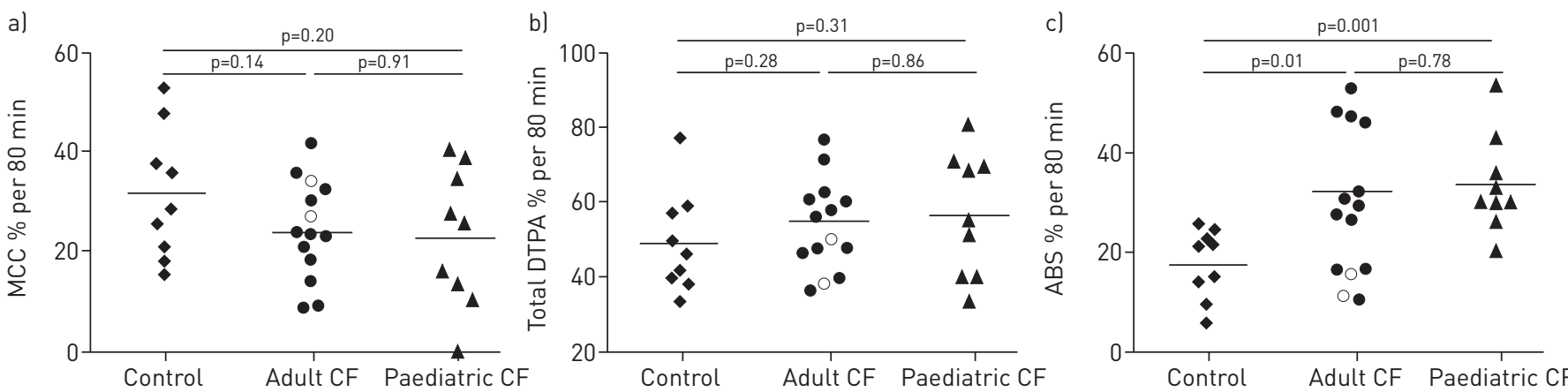

FIGURE 3 a) Mucociliary clearance (MCC), b) total diethylene triamine penta-acetic acid (DTPA) clearance and c) DTPA absorption (ABS) in healthy volunteers $(n=9)$, and adult $(n=12)$ and paediatric cystic fibrosis $(C F)(n=9)$ subjects following nebulised isotonic saline treatment. Horizontal lines represent the mean clearance rate for each study group. Open circles represent G551D subjects utilizing ivacaftor (not included in statistical analysis). 
TABLE 2 Central lung zone and area above the curve analysis

Controls

Paediatric CF

Adult CF

IS

HS

Central MCC \%
Central DTPA \%
Central ABS \%
AACTc
AACIn
AACABS

$$
\begin{gathered}
36.2 \pm 18.0 \\
45.4 \pm 16.6 \\
9.2 \pm 10.0 \\
18.6 \pm 9.4 \\
25.9 \pm 9.6 \\
7.3 \pm 3.5
\end{gathered}
$$

$35.2 \pm 18.0$
$57.1 \pm 20.6$
$21.9 \pm 5.0^{* *}$
$14.5 \pm 8.8$
$30.6 \pm 11.4$
$16.1 \pm 7.7^{* *}$

$32.7 \pm 13.5$

$56.0+19.0$

$23.3 \pm 13.0^{*}$

$14.3 \pm 6.3$

$30.5+10.3$

$16.2 \pm 8.8^{* *}$
$45.7 \pm 23.7$

$62.8 \pm 21.7$

$17.1 \pm 12.2^{\#}$

$26.8 \pm 10.8^{\# \#}$

$38.8+7.7^{\#}$

$12.0 \pm 6.8^{\# \#}$

Data are presented as mean \pm SD. CF: cystic fibrosis; IS: isotonic saline; HS: hypertonic saline; MCC: mucociliary clearance; DTPA: diethylene triamine penta-acetic acid; ABS: DTPA absorption; AACTc: area above the technetium- $99 \mathrm{~m}$-sulfur colloid retention curve; AACIn: area above the ${ }^{111}$ In-DTPA retention curve; AACABS: area above the ABS curve. ${ }^{*}: p<0.05$ versus controls; ${ }^{* *}: p<0.01$ versus controls; ${ }^{\#}$ : $<<0.05$ versus adult CF subjects with IS treatment; ${ }^{\# \#: ~} \mathrm{p}<0.01$ versus adult CF subjects with IS treatment.

an increase in total DTPA clearance with hypertonic saline treatment $(\mathrm{p}=0.118$ ) (fig. 4c). AAC analysis showed that AACTc and AACIn were significantly increased $(\mathrm{p}=0.001$ and $\mathrm{p}=0.04$, respectively), and AACABS was significantly decreased $(p=0.008)$ following hypertonic saline treatment. Baseline deposition pattern $(\mathrm{C} / \mathrm{P})$ did not differ between treatment days $(2.4 \pm 0.5$ versus $2.3 \pm 0.5 ; \mathrm{p}=0.69)$ and there was no correlation between the per cent change in ABS and per cent change in MCC between treatment days. Baseline MCC values were increased and ABS values were decreased in the two adult ivacaftor subjects compared with average values in the adult CF group. Following hypertonic saline treatment, one ivacaftor subject showed a strong increase in MCC while the other showed a moderate decrease. Both subjects showed a moderate decrease in ABS with hypertonic saline treatment.

\section{Relationship of image-derived measurements to clinical variables and deposition pattern}

Image-derived retention measurements were compared to pulmonary function data and other clinical variables using linear regression analysis. For all subject groups, neither ABS or AACABS correlated with forced expiratory volume in $1 \mathrm{~s}$ (FEV1), forced expiratory flow at $25-75 \%$ of forced vital capacity (FEF25-75\%), age or sweat chloride concentration with the exception that in the paediatric CF group, ABS and AACABS increased significantly with age $(\mathrm{p}=0.03$ and $\mathrm{p}=0.05$, respectively). Age did not correlate with MCC in the paediatric CF group but MCC did increase with FEV1 $(p=0.05)$ and FEF25-75\% $(p=0.05)$. No correlations were found between MCC or AACTc and pulmonary function variables in the adult subject groups.

In all groups combined, MCC correlated with total DTPA clearance $(p=0.01)$ demonstrating the effect of MCC on the small molecule probe. There were no correlations between C/P and ABS, MCC or total DTPA clearance, or with the AAC values for these variables. Initial indium dose did not correlate with MCC, ABS or total DTPA clearance. Similarly, there were no correlations between initial technetium dose and any clearance parameters.
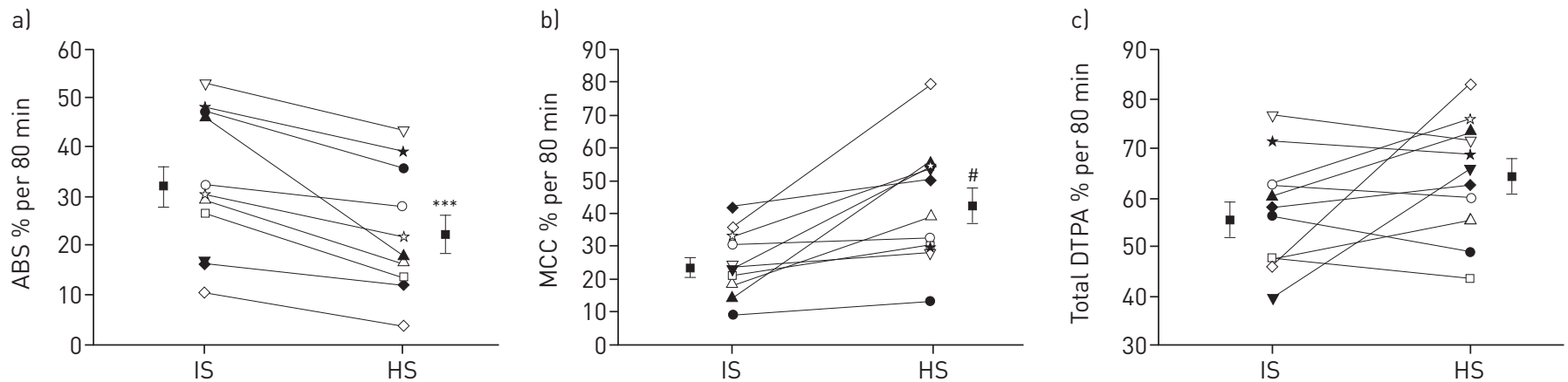

FIGURE 4 The effects of nebulised hypertonic saline (HS) treatment in individual cystic fibrosis adult subjects. a) Indium-111-diethylene triamine penta-acetic acid (DTPA) absorption (ABS) was decreased in every subject after inhalation of HS treatment. b) Technetium-99m-sulfur colloid clearance was increased by HS treatment in 11 out of 11 subjects. c) Total ${ }^{111}$ In-DTPA clearance was increased by HS treatment in six out of 11 subjects $(p=0.118)$. Paired $t$-test was used. Data are presented as mean \pm SEM. IS: isotonic saline; MCC: mucociliary clearance. ${ }^{* * *}: \mathrm{p}<0.001$ versus IS; ${ }^{*}: \mathrm{p}=0.003$ versus IS. 

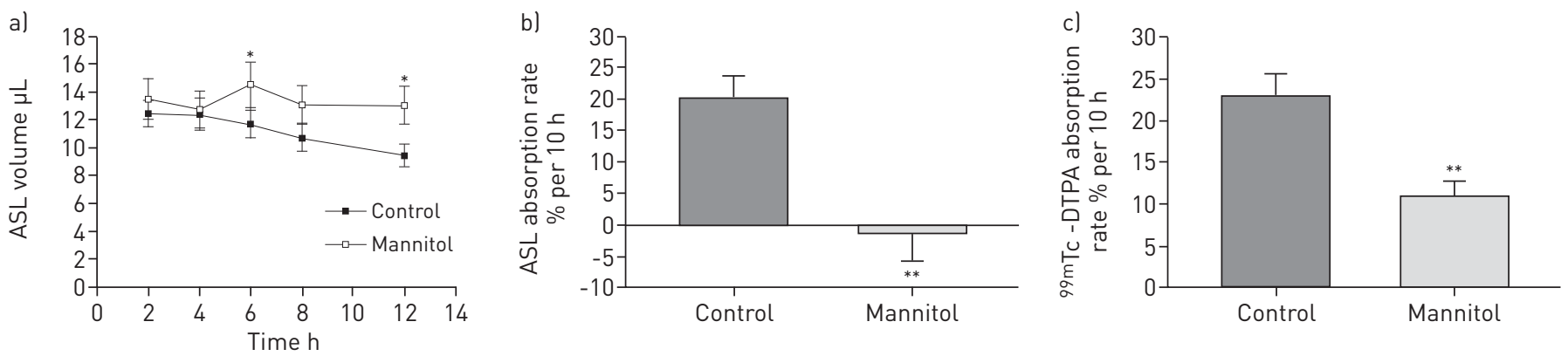

FIGURE 5 Comparison of airway surface liquid (ASL) volume and diethylene triamine penta-acetic acid (DTPA) absorption after addition of isotonic saline versus iso-osmolar solution of mannitol in water. a) ASL volume following apical volume expansion with either $10 \mu \mathrm{L}$ isotonic saline (control) or $300 \mathrm{mM}$ mannitol in water on differentiated cystic fibrosis human bronchial epithelial cell cultures. b) The per cent ASL absorption rate from 2 to $12 \mathrm{~h}$ after apical addition of isotonic saline or mannitol in water. c) The per cent ${ }^{99 \mathrm{~m}} \mathrm{Tc}$-DTPA absorption rate over the same 10-h period after apical addition of isotonic saline or mannitol in water. Data are presented as mean \pm SEM of 18 cultures from three different tissue donors. ${ }^{*}: \mathrm{p}<0.05$ versus control; ${ }^{* *}: \mathrm{p}<0.01$ versus control.

\section{The contribution of transepithelial fluid flow to DTPA absorption}

As shown in figure 5a, there was continual liquid absorption from 2 to $12 \mathrm{~h}$ following the bolus addition of $10 \mu \mathrm{L}$ isotonic saline, absorbing at a rate of $20.1 \pm 3.6 \%$ per $10 \mathrm{~h}$. Conversely, a static ASL volume was observed after the apical addition of the mannitol solution, with a net absorption of $-1.2 \pm 4.4 \%$ per $10 \mathrm{~h}$ $(\mathrm{p}<0.001)$ (fig. 5b). DTPA was absorbed at a rate of $23.1 \pm 2.6 \%$ per $10 \mathrm{~h}$ when delivered in normal saline and $10.4 \pm 1.7 \%$ per $10 \mathrm{~h}$ when delivered in the iso-osmolar solution of mannitol in water $(\mathrm{p}<0.001)$ (fig. 5c), indicating that approximately one-half of DTPA absorption is associated with transepithelial fluid transport with the remainder presumably being associated with diffusion.

\section{The effect of accumulated mucus on ASL volume and DTPA absorption}

As shown in figure $6 \mathrm{a}$, the rate of ASL volume absorption was significantly decreased when exogenous mucus was applied to the apical cell surface $(8.6 \pm 3.8 \%$ versus $35.3 \pm 2.4 \%$ per $12 \mathrm{~h}$ for control, $\mathrm{p}<0.001)$ and significantly increased by a transepithelial osmotic gradient generated by basolateral mannitol $(54.4 \pm 2.6 \%$ per $12 \mathrm{~h}, \mathrm{p}<0.001$ versus control). In the presence of both exogenous mucus and the transepithelial osmotic gradient, the rate of ASL volume absorption remained significantly elevated $(49.3 \pm 1.5 \%$ per $12 \mathrm{~h}, \mathrm{p}<0.03$ versus control). DTPA absorption mirrored the response of ASL volume absorption for each of the conditions described above. The rate of DTPA absorption was significantly decreased by exogenous mucus $(13.3 \pm 3.1 \%$ versus $37.7 \pm 2.6 \%$ per $12 \mathrm{~h}, \mathrm{p}<0.001)$ and was significantly increased by the transepithelial osmotic gradient $(93.7 \pm 0.6 \%$ per $12 \mathrm{~h}, \mathrm{p}<0.001$ versus control). DTPA absorption in the presence of both mucus and the osmotic gradient was $90.6 \pm 1.2 \%$ per $12 \mathrm{~h}(\mathrm{p}<0.03$ versus control). These data reveal that the impeding effects of apical mucus on the rates of both DTPA and ASL volume absorption become negligible in the presence of an osmotic gradient.
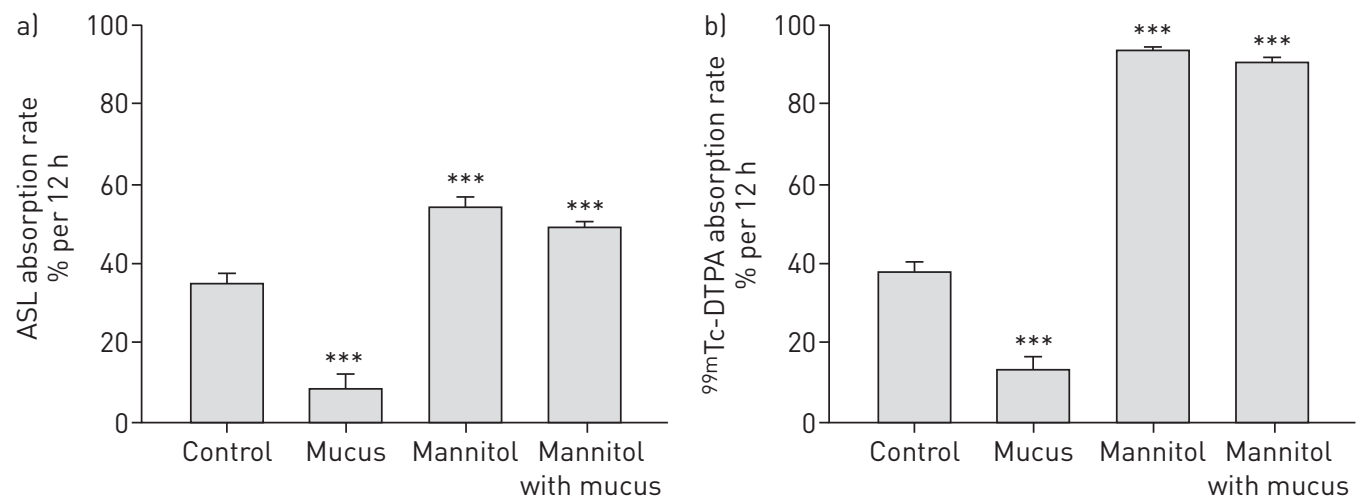

FIGURE 6 The effect of accumulated mucus on airway surface liquid (ASL) and diethylene triamine penta-acetic acid (DTPA) absorption in cystic fibrosis (CF) human bronchial epithelial (HBE) cell cultures. a) ASL volume absorption represented as a per cent change at $12 \mathrm{~h}$ in the absence or presence of mucus (10 $\mu \mathrm{L}$, sampled from non-CF HBE cells), hyperosmotic basolateral growth medium (normal medium plus $300 \mathrm{mM}$ mannitol) or the combination of both. b) ${ }^{99 \mathrm{~m}} \mathrm{Tc}$-DTPA absorption represented as a per cent change at $12 \mathrm{~h}$ in the absence or presence of mucus, hyperosmotic basolateral media or both. Data are presented as mean \pm SEM of 12 cultures from two different tissue donors. ${ }^{* * *}$ : $\mathrm{p}<0.001$ versus control. 


\section{Discussion}

Airway liquid hyperabsorption is a key contributor to the impairment of MCC in CF. Measuring changes in airway liquid absorption through functional imaging methods may allow for rapid screening of medications designed to restore ASL volume, such as inhaled osmotics, sodium channel blockers, and CFTR correctors and potentiators. In the current study, MCC and DTPA absorption were measured in vivo using dualisotope $\left({ }^{99 \mathrm{~m}} \mathrm{Tc}\right.$-SC and ${ }^{111} \mathrm{In}$-DTPA) imaging. While statistically significant differences in baseline MCC were not detected between study groups, DTPA absorption was found to be increased in CF patients, consistent with our previous in vivo [11] and in vitro [10] results. These findings were similar in central zone measurements and when data were analysed using the AAC. Interestingly, the majority of paediatric CF patients who demonstrated airway hyperabsorption of DTPA had well-maintained pulmonary function, implying that DTPA absorption is linked to a basic defect of CF lung disease that precedes the decline in FEV1. We also demonstrated the acute effects of inhaled hypertonic saline by showing that it significantly increases MCC and decreases DTPA absorption in adult CF patients, a result consistent with its mechanism of action of hydrating the airway surface.

The underlying mechanisms contributing to airway DTPA absorption rates probably involve the net transport of sodium across the epithelium mediated by the epithelial sodium channel (ENaC) $[16,20]$. In $\mathrm{CF}, \mathrm{ENaC}$ becomes dysregulated, leading to the hyperabsorption of sodium [20]. The transepithelial movement of water that occurs in response to the skewed sodium gradient is a likely driving force for the heightened DTPA absorption measurement in our CF cohorts. Future studies could investigate changes in DTPA absorption with direct pharmacological blockers of ENaC to begin to probe this mechanism.

Baseline DTPA absorption measurements in adult CF subjects demonstrated significant intersubject variability compared with paediatric CF subjects and healthy controls. In light of previous work that showed that mucus possesses binding sites for ${ }^{99 \mathrm{~m}} \mathrm{Tc}$-DTPA [19], we posited that the variability observed in the adult subjects may be partially attributable to different volumes of accumulated mucus between subjects. This is supported by the finding that apical mucus reduced the rate of ASL and DTPA absorption in cultured primary HBE cells. However, the rate of ASL volume and DTPA absorption increased in response to a basolateral osmotic gradient in the presence or absence of apical mucus. This finding argues against DTPA binding to mucus, instead favouring a mechanism whereby mucus acts as a resistor to transepithelial fluid flow. We conclude that the intersubject variability in baseline DTPA absorption among the adult CF subjects may be at least partly attributable to differential amounts of accumulated mucus.

While the two-dimensional planar imaging used in this study is able to provide high temporal resolution, it does not allow for discrimination between airway and alveolar compartments. In the peripheral lung, DTPA clearance is highly sensitive to changes in permeability mediated by inflammatory stimuli such as cigarette smoke [21]. In CF, lung disease typically progresses from the airways; however, inflammation in alveoli has been described [22], as have CFTR-related differences in alveolar fluid transport [23]. Measurements of absorption made in this study will probably include some contribution from alveoli; however, we do not anticipate that these effects will dominate the measurement due to the delivery techniques used to target aerosol deposition to the airways. The high $\mathrm{C} / \mathrm{P}$ dose ratios and MCC rates measured here indicate general success in this strategy.

DTPA absorption measurements will probably be a function of both transepithelial liquid transport and paracellular permeability associated with physical properties of airway epithelial cells. Our in vitro studies demonstrated that liquid absorbing through the epithelium accounted for about half of the total DTPA absorbed, with simple diffusion probably accounting for the rest (fig. 5). It has been previously documented that CF HBE cell cultures exhibited lower transepithelial resistance (TER) values, a measurement related to paracellular permeability [24]. However, studies performed in our laboratory demonstrated no relationship between TER values and DTPA absorption rates in CF and non-CF HBE cell cultures [10]. While we cannot exclude the possibility that differences in baseline absorption between CF and non-CF subjects may be the result of differences in airway epithelial integrity [25, 26], the consistent and significant effects of osmotic therapies on DTPA absorption demonstrated both in vitro and in vivo favour a mechanism linked to liquid transport.

Some previous imaging studies have shown significant differences in whole-lung MCC between CF and non-CF subjects $[15,27]$, while others have not [6]. Our results showed that baseline MCC in CF subjects was not significantly decreased compared with control subjects. It is possible that the aerosol distribution pattern may not be optimised to expose MCC deficiencies between non-CF and CF subjects during nonexacerbation periods, or that the duration of the measurement was too short to detect a difference. Additionally, our study was powered primarily to detect therapy-associated changes in measured values through paired comparisons. We estimate that we could detect an $\sim 8 \%$ therapy-associated change in MCC with 12 subjects, for example. However, our study was probably not sufficiently powered to 
detect a difference between baseline measurements of MCC through unpaired comparisons between the CF and non-CF groups. We estimate that only baseline differences $\geqslant 17 \%$ would be detectable with 12 subjects.

Three CF patients with the G551D mutation were included in our study, two of whom were on ivacaftor therapy. DTPA absorption rates in the treated subjects were substantially lower than the CF average, while the untreated subject demonstrated absorption above the mean rate of the CF group (52.9\% per $80 \mathrm{~min}$ ). The rate of MCC in the untreated subject was within $2 \%$ of the average of the CF group, while the treated subjects had MCC rates substantially higher than the CF average (34.3\% and $27.2 \%$ per 80 min). While this observation is interesting, it is impossible to link ivacaftor treatment with DTPA absorption response without establishing a baseline and studying more subjects.

In conclusion, DTPA absorption provides a quantifiable marker of immediate response to treatments that influence airway hydration in CF patients and may accelerate CF therapy development. DTPA absorption was increased in paediatric CF patients with normal spirometry values, suggesting that our imaging test elucidates the basic defect in airway hydration prior to the development of abnormal spirometry. We also demonstrated significant decreases in DTPA absorption rate after treatment with inhaled hypertonic saline. Though multiple factors may affect DTPA absorption across airway epithelia, our in vitro and in vivo results demonstrate a significant dependence on transepithelial fluid movement.

\section{Acknowledgements}

The authors wish to thank Adrienne DeRicco, Elizabeth Hartigan and Sandra Hurban (all Children's Hospital of Pittsburgh, University of Pittsburgh Medical Center, Pittsburgh, PA, USA) for their assistance in performing these studies.

\section{References}

Boucher RC. New concepts of the pathogenesis of cystic fibrosis lung disease. Eur Respir J 2004; 23: 146-158.

2 Eckford PD, Li C, Ramjeesingh M, et al. Cystic fibrosis transmembrane conductance regulator (CFTR) potentiator VX-770 (ivacaftor) opens the defective channel gate of mutant CFTR in a phosphorylation-dependent but ATPindependent manner. J Biol Chem 2012; 287: 36639-36649.

3 McPhail GL, Clancy JP. Ivacaftor: the first therapy acting on the primary cause of cystic fibrosis. Drugs Today (Barc) 2013; 49: 253-260.

4 Fajac I, Hubert D, Bienvenu T, et al. Relationships between nasal potential difference and respiratory function in adults with cystic fibrosis. Eur Respir J 1998; 12: 1295-1300.

5 Bennett WD, Ilowite JS. Dual pathway clearance of ${ }^{99 \mathrm{~m}}$ Tc-DTPA from the bronchial mucosa. Am Rev Respir Dis 1989; 139: 1132-1138.

6 Donaldson SH, Bennett WD, Zeman KL, et al. Mucus clearance and lung function in cystic fibrosis with hypertonic saline. N Engl J Med 2006; 354: 241-250.

7 Robinson M, Regnis JA, Bailey DL, et al. Effect of hypertonic saline, amiloride, and cough on mucociliary clearance in patients with cystic fibrosis. Am J Respir Crit Care Med 1996; 153: 1503-1509.

8 Blouquit S, Regnier A, Dannhoffer L, et al. Ion and fluid transport properties of small airways in cystic fibrosis. Am J Respir Crit Care Med 2006; 174: 299-305.

9 Chambers LA, Rollins BM, Tarran R. Liquid movement across the surface epithelium of large airways. Respir Physiol Neurobiol 2007; 159: 256-270.

10 Corcoran TE, Thomas KM, Brown S, et al. Liquid hyper-absorption as a cause of increased DTPA clearance in the cystic fibrosis airway. EJNMMI Res 2013; 3: 14.

11 Corcoran TE, Thomas KM, Myerburg MM, et al. Absorptive clearance of DTPA as an aerosol-based biomarker in the cystic fibrosis airway. Eur Respir J 2010; 35: 781-786.

12 Donaldson SH, Corcoran TE, Laube BL, et al. Mucociliary clearance as an outcome measure for cystic fibrosis clinical research. Proc Am Thorac Soc 2007; 4: 399-405.

13 Bennett WD, Laube BL, Corcoran T, et al. Multisite comparison of mucociliary and cough clearance measures using standardized methods. J Aerosol Med Pulm Drug Deliv 2013; 26: 157-164.

14 Robinson M, Daviskas E, Eberl S, et al. The effect of inhaled mannitol on bronchial mucus clearance in cystic fibrosis patients: a pilot study. Eur Respir J 1999; 14: 678-685.

15 Regnis JA, Robinson M, Bailey DL, et al. Mucociliary clearance in patients with cystic fibrosis and in normal subjects. Am J Respir Crit Care Med 1994; 150: 66-71.

16 Myerburg MM, Harvey PR, Heidrich EM, et al. Acute regulation of the epithelial sodium channel in airway epithelia by proteases and trafficking. Am J Respir Cell Mol Biol 2010; 43: 712-719.

17 Hurt K, Bilton D. Inhaled mannitol for the treatment of cystic fibrosis. Exp Rev Respir Med 2012; 6: 19-26.

18 Harvey PR, Tarran R, Garoff S, et al. Measurement of the airway surface liquid volume with simple light refraction microscopy. Am J Respir Cell Mol Biol 2011; 45: 592-599.

19 Cheema MS, Groth S, Marriott C. Binding and diffusion characteristics of ${ }^{14} \mathrm{C}$ EDTA and ${ }^{99 \mathrm{~m}} \mathrm{Tc}$ DTPA in respiratory tract mucus glycoprotein from patients with chronic bronchitis. Thorax 1988; 43: 669-673.

20 Donaldson SH, Boucher RC. Sodium channels and cystic fibrosis. Chest 2007; 132: 1631-1636.

21 Nolop KB, Maxwell DL, Fleming JS, et al. A comparison of ${ }^{99 \mathrm{~m}}$ Tc-DTPA and ${ }^{113 \mathrm{~m}}$ In-DTPA aerosol clearances in humans. Effects of smoking, hyperinflation, and in vitro oxidation. Am Rev Respir Dis 1987; 136: 1112-1116.

22 Ulrich M, Worlitzsch D, Viglio S, et al. Alveolar inflammation in cystic fibrosis. J Cyst Fibros 2010; 9: $217-227$.

23 Li X, Comellas AP, Karp PH, et al. CFTR is required for maximal transepithelial liquid transport in pig alveolar epithelia. AJP Lung Cell Mol Physiol 2012; 303: L152-L160. 
24 Devor DC, Pilewski JM. UTP inhibits $\mathrm{Na}^{+}$absorption in wild-type and $\triangle \mathrm{F} 508$ CFTR-expressing human bronchial epithelia. Am J Physiol 1999; 276: C827-C837.

25 Nilsson HE, Dragomir A, Lazorova L, et al. CFTR and tight junctions in cultured bronchial epithelial cells. Exp Mol Pathol 2010; 88: 118-127.

26 LeSimple P, Liao J, Robert R, et al. Cystic fibrosis transmembrane conductance regulator trafficking modulates the barrier function of airway epithelial cell monolayers. J Physiol 2010; 588: 1195-1209.

27 Robinson M, Eberl S, Tomlinson C, et al. Regional mucociliary clearance in patients with cystic fibrosis. J Aerosol Med 2000; 13: 73-86. 